\title{
ABSOLUTE ENERGY DISTRIBUTION IN RHO CASSIOPEIAE
}

S.C. Joshi and B.s. Rautela

Uttar Pradesh State Observatory, Nanora Peak,

Naini Tal, India.

\begin{abstract}
The continuous energy distribution of p Cas obtained in the wavelength range 340 - $710 \mathrm{~nm}$ has been compared with the energy distribution of 8 CMa in the same wavelength range. The circumetellar shell associated with $p$ Cas seens to modify the continuum of the star appreciably. Variations in the Balmer emission of $p$ Cas taking place within a day are noticed.

Detalls of this paper will shortly appear in the Monthly Notices of the Royal Astronomical society.
\end{abstract}

